\title{
Mesoscopic Simulations of Biological Membranes
}

B. Smit ${ }^{1,2}$, M. Kranenburg ${ }^{2}$, M. M. Sperotto ${ }^{3}$, and M. Venturoli ${ }^{4}$

${ }^{1}$ Centre Européen de Calcul Atomique et Moleculaire (Cecam), Ecole Normale Supérieure, 46 Allée d'Italie, 69007 Lyon, France

b.smit@cecam.fr

2 Van 't Hoff Institute for Molecular Sciences, University of Amsterdam, Nieuwe Achtergracht 166, 1018 WV Amsterdam, The Netherlands

marieke@science.uva.nl

3 Biocentrum, The Technical University of Denmark, Kgs. Lyngby, Denmark maria@cbs.dtu.dk

${ }^{4}$ CCS, University College, C. Ingold Labs, 20 Gordon St., London WC1H 0AJ, U.K.

m.venturoli@ucl.ac.uk

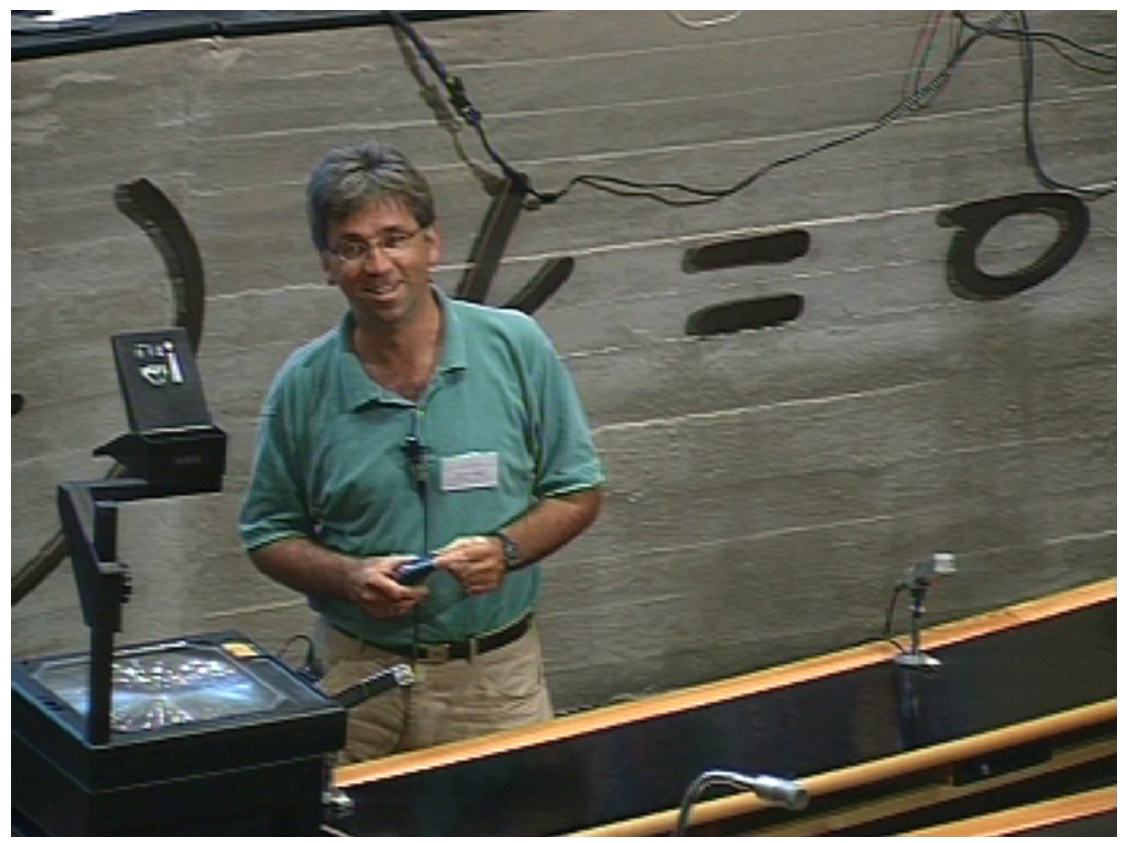

Berend Smit

B. Smit et al.: Mesoscopic Simulations of Biological Membranes, Lect. Notes Phys. 704, 259286 (2006) 


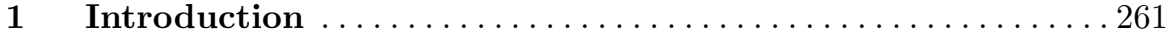

2 Computer Simulations Aspects ................... 263

2.1 Dissipative Particle Dynamics . . . . . . . . . . . . . . . . . . 263

2.2 Surface Tension in Lipid Bilayers ......................... 264

3 Phase Behavior of Coarse-Grained Lipid Bilayers ........ 266

3.1 Single-Tail Lipid Bilayers .......................... 267

3.2 Double-Tail Lipid Bilayers ............................. 272

4 Perturbations of the Membrane Structure..............2274

4.1 Effect of Alcohol . . . . . . . . . . . . . . . . . . . . . . . . . 275

4.2 Bilayers with Transmembrane Proteins . .................. 278

$5 \quad$ Concluding Remarks ............................. 280

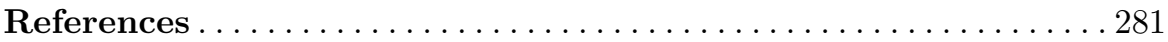




\section{Introduction}

A common structure found in all cells and theirs inner organelles is the membrane. Biological membranes act as semi-permeable barriers, allowing a selected passage of small molecules or ions. Biomembranes are constituted of a lipid matrix in which molecules such as proteins or cholesterol are embedded or attached. Lipids are amphiphilic molecules, i.e. molecules constituted of an hydrophilic polar headgroup, which is water soluble, and hydrophobic tails, which are water insoluble. The lipid matrix is formed by the non-covalent self-assembly of two lipid monolayers made of a variety of lipid types.

The combination of hydrophobic and hydrophilic groups in the same molecule is a key factor for the assembly of lipids into supra-molecular aggregates, such as micelles or vesicles, the latter being the templates for the cell membranes. Due to the hydrophobic effect $[1,2]$ membrane lipids assemble in such a way that their hydrophobic part is excluded from a direct contact with the water environment, while the hydrophilic or polar parts are in direct contact with the water. The resulting pseudo two-dimensional system (Fig. 1) is a fluid structure where the molecules may diffuse in the membrane plane, may flip-flop from one monolayer to another, or may even move out of the system.

Biological membranes are not only inert walls, but complex, organized, dynamic, and highly cooperative structures whose physical properties are important regulators of vital biological functions ranging from cytosis and nerve processes, to transport of energy and matter [3].

To relate the biomembrane structure and dynamics to their biological function - the ultimate goal of biomembrane science- is often necessary to consider simpler systems. Lipid bilayers composed of one or two lipid species, and with embedded proteins or natural or artificial peptides, provide a model system for biological membranes. Understanding the physics of such simplified softcondensed matter systems can yield insight into biological membrane functions.

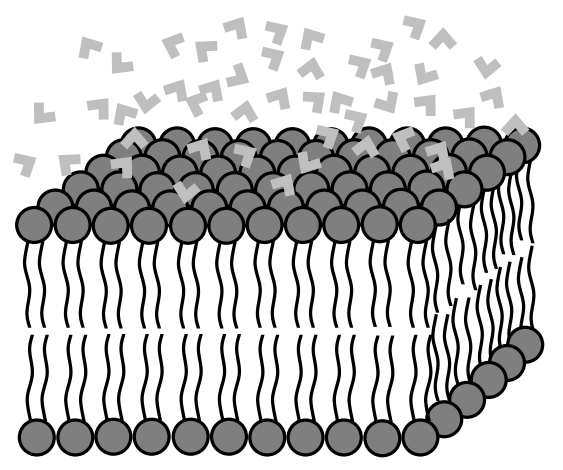

aqueous environment

polar heads

hydrocarbon tails

Fig. 1. Schematic representation of a lipid bilayer 
Because of the many degrees of freedom involved, the processes that take place even in model biomembranes occur over a wide range of time and length scales [4]. The typical time and length scales of the processes under investigation do pose limitations on the details of the model. Often, this necessity follows the fact that some theoretical methods are limited in their applicability by the long computational times needed to compute statistical quantities.

Molecular Dynamics (MD) simulation methods on atomistic detailed models have been used to study the structural and dynamic properties of membranes [5], the self-assembly of phospholipids into bilayers [6], as well as the interactions of membrane proteins or other molecules with the lipid bilayer [7-12]. MD simulations can provide detailed informations about the phenomena that occur in biomembrane systems, although at the nanoscopic level and on a nanosecond time-scale. Many membrane processes happen though at the mesoscopic length and time scale, i.e. $>1-1000 \mathrm{~nm}$, ns, respectively, and involve the collective nature of the system.

An alternative modeling approach consists in neglecting most of the molecular details of the system. The resulting lattice [13,14], interfacial [15], or phenomenological models [16-18], are computationally very efficient, and can give us insight into the physical properties of reconstituted membranes $[19,20]$. However, with the help of these models, it is difficult to study some structural and conformational properties of the system, which derive from some molecular details, and which results from its cooperative behavior. To overcome this difficulty, we have developed a model for lipid systems which can be seen as an intermediate between the all-atom models and the models briefly just mentioned. This mesoscopic model considers a system of "particles", or "beads", in which each "particle" represents a complex molecular component of the system whose details are not important to the process under investigation. Models with simplified interactions between the "beads" are called coarse-grain (CG) models. In Fig. 2 the chemical structure of the phospholipid dimyristoylphosphatidylcholine (DMPC) and its CG representation are shown. In the recent years, CG models have been developed to study the phase equilibria of biomembrane-like systems at the mesoscopic level, and both MD

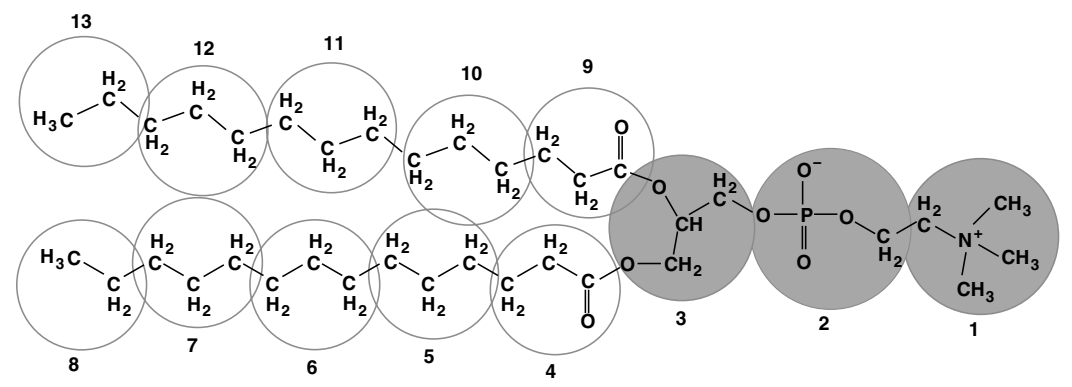

Fig. 2. The atomistic representation of DMPC and its corresponding coarse-grained model 
and Monte Carlo (MC) simulation methods were used on such models [21-25]. In this contribution we summarize some of the results of our simulations. A more complete account can be found in [26-34].

\section{Computer Simulations Aspects}

\subsection{Dissipative Particle Dynamics}

The simulations presented in this contribution are carried out with Dissipative Particle Dynamics (DPD) [35]. By combining several aspects of Molecular Dynamics and lattice-gas automata, DPD captures hydrodynamic time and length scales much larger than can be reached with the first method and it avoids the lattice artifacts of the latter method. One of the most attractive features of DPD is the versatility in simulating complex fluids. A DPD particle represents a "fluid package" or a cluster of atoms, that moves according to the Newton's equations of motion, interacting with other particles through a dissipative, a random, and a conservative force. By changing the conservative force between different types of particles a fluid can be made "complex". See $[36,37]$ for nice reviews on DPD and its applications. Recently, DPD has also been used to study the behavior of a lipid bilayer [24, 26, 38, 39].

A DPD particle represents the center of mass of a cluster of atoms. The particles interact via a force consisting of three contributions, all of them pairwise additive. The total force on a particle $i$ consists of a dissipation force $\mathbf{F}^{D}$, a random force $\mathbf{F}^{R}$, and a conservative force $\mathbf{F}^{C}$, and can then be written as the sum of these forces $[35,40]$ :

$$
\mathbf{f}_{i}=\sum_{i \neq j}\left(\mathbf{F}_{i j}^{D}+\mathbf{F}_{i j}^{R}+\mathbf{F}_{i j}^{C}\right)
$$

The first two forces in equation 1 are of the form:

$$
\begin{aligned}
& \mathbf{F}_{i j}^{D}=-\eta w^{D}\left(r_{i j}\right)\left(\hat{\mathbf{r}}_{i j} \cdot \mathbf{v}_{i j}\right) \hat{\mathbf{r}}_{i j} \\
& \mathbf{F}_{i j}^{R}=\sigma w^{R}\left(r_{i j}\right) \zeta_{i j} \hat{\mathbf{r}}_{i j}
\end{aligned}
$$

where $\mathbf{r}_{i j}=\mathbf{r}_{i}-\mathbf{r}_{j}$ and $\mathbf{v}_{i j}=\mathbf{v}_{i}-\mathbf{v}_{j}$, with $\mathbf{r}_{i}$ and $\mathbf{v}_{i}$ representing the position and the velocity of particle $i$, respectively. $\hat{\mathbf{r}}_{i j}$ is the unit vector, $\eta$ is the friction coefficient, $\sigma$ the noise amplitude, and $\zeta_{i j}$ a random number taken from a uniform distribution, which is independent for each pair of particles. The combined effect of these two forces is a thermostat, which conserves (angular) momentum, and hence gives the correct hydrodynamics at sufficiently long time and length scales.

Español and Warren [41] have shown that the equilibrium distribution of the system is the Gibbs-Boltzmann distribution if the weight functions and coefficients of the drag and the random force satisfy: 


$$
\begin{aligned}
w^{D}(r) & =\left[w^{R}(r)\right]^{2} \\
\sigma^{2} & =2 \eta k_{B} T
\end{aligned}
$$

The weight function $w^{R}(r)$ is chosen as

$$
w^{R}(r)= \begin{cases}\left(1-r / r_{c}\right) & \left(r<r_{c}\right) \\ 0 & \left(r \geq r_{c}\right)\end{cases}
$$

where $r_{c}$ is the cut-off radius, which gives the extent of the interaction range. In this case, all forces assume the same functional dependence on the interparticle distance $\mathbf{r}_{i j}$ as the conservative force $\mathbf{F}_{i j}^{C}$. Español and Warren have also shown that a DPD Hamiltonian can be defined and the existence of a Hamiltonian implies that only the conservative part of the force (or better, the potential $U_{C}$ related to it: $\mathbf{F}^{C}=-\nabla U_{C}$ ), determines the equilibrium averages of the system observables. Furthermore, as shown by Willemsen et al. in [42], this implies that DPD can be combined with Monte-Carlo (MC) methods.

Most DPD simulation use soft-repulsive interactions of the form

$$
\mathbf{F}_{i j}^{C}= \begin{cases}a_{i j}\left(1-r_{i j} / r_{c}\right) \hat{\mathbf{r}}_{i j} & \left(r_{i j}<r_{c}\right) \\ 0 & \left(r_{i j} \geq r_{c}\right)\end{cases}
$$

where the coefficient $a_{i j}>0$ is a parameter expressing the maximum repulsion strength. Alltough any form of conservative interactions can be used in a DPD simulation, these soft-repulsive interactions are often refer to as the DPD model.

\subsection{Surface Tension in Lipid Bilayers}

Lipid bilayers are self-assembled structures, which are not constrained by the total area, and hence will adopt a conformation that will have the lowest free energy. Since the thermodynamic definition of surface tension is the derivative of the free energy with respect to the area of the interface [43], for a unconstrained bilayer the free energy minimum will be a tensionless state [44]. Experimental results on unilamellar vesicles have also indicated that bilayers are in a stress free state [45]. In molecular simulation, for both self-assembled and pre-assembled membranes, a fixed number of lipid molecules and a fixed area are usually combined with periodic boundary conditions. The periodic boundary conditions correspond to an infinitely large membrane, but the fixed size of the simulation box, and the fixed number of lipids at the interface, impose a constraint on the bilayer area which results in a finite surface tension. Although the constraint on the fixed area can be released by performing simulations of membranes at constant pressure or constant surface tension [46,47], it is an important -and still open-question which value of the surface tension should be used in simulations to reproduce the state and the area per lipid of a real membrane. 
In their molecular dynamics simulations Feller and Pastor [48,49] observed that a tensionless state did not reproduce the experimental value of the area per lipid. They explained this result by considering that, since the typical undulations and and out-of-plane fluctuations of a macroscopic membrane do not develop in a small patch of a membrane (theirs was composed of 36 lipids for monolayer), a positive surface tension (stretching) must be imposed in order to compensate for the suppressed undulations, and hence to recover the experimental values of the area per lipid. Recently, Marrink and Mark [50] investigated the system size dependence of the surface tension in membrane patches ranging from 200 to 1800 lipids, simulated for times up to 40ns. Their calculations show that, in a stressed membrane, the surface tension is size dependent, i.e. it drops if the system size is increased (at fixed area per lipid), which is in agreement with the results by Feller and Pastor. On the other hand, their results show that at zero stress simulation conditions the equilibrium does not depend on the system size. Marrink and Mark then concluded that simulations at zero surface tension correctly reproduce the experimental surface areas for a stress free membrane.

Simulations at constant surface tension have been introduced by Chiu et al. in [46]. A constant surface tension ensemble $(N V \gamma)$ has been considered in literature and the corresponding equations of motion for Molecular Dynamics simulations have been derived [47], and applied to the simulation of phospholipid bilayers $[49,51-55]$. Alternatively, to ensure a tensionless state, Goetz and Lipowsky [21] performed several simulations to determine the area per lipid that gives a state of zero tension. An alternative approach it to combine DPD or MD with a Monte Carlo move that imposes a given value for the surface tension [26]. The advantage of these constant surface tension scheme is that one can directly observe phase transitions in which the area per lipid changes.

Consider a simulation box (see Fig. 3) with edges $L_{x}=L_{y}=L_{\|}$parallel to the interface ( $x y$ plane), and $L_{z}=L_{\perp}$ perpendicular to the interface $(z$ axis), so that the system volume is $V=L_{\perp} L_{\|}^{2}$ and the area of the interface $A=L_{\|}^{2}$. We define a transformation of the box sizes which changes the area and the height but keeps the volume constant. Such a transformation can be written in the form

$$
\begin{gathered}
L_{\|}^{\prime}=\lambda L_{\|} \\
L_{\perp}^{\prime}=\frac{1}{\lambda^{2}} L_{\perp}
\end{gathered}
$$

where $\lambda$ is the parameter of the transformation. By changing $\lambda$, the above expression generates a transformation of coordinates which preserves the total volume of the system, hence no work against the external pressure is performed. In a MC move an attempt of changing the parameter $\lambda$ is then accepted with a probability 


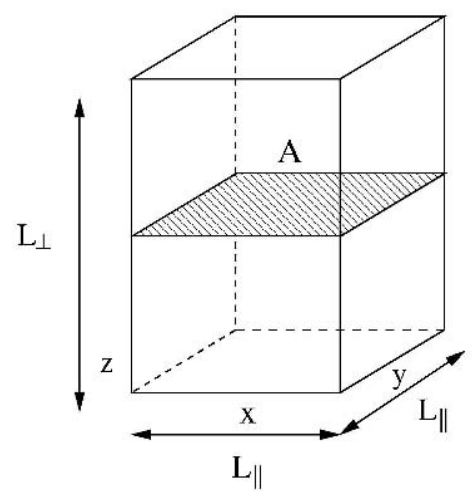

Fig. 3. Schematic representation of a simulation box for a system with a flat interface parallel to the $x y$-plane. The area of the interface is $A=L_{\|}^{2}$ and the box dimension perpendicular to the interface (z-axis) is $L_{\perp}$

$$
\operatorname{acc}\left(\lambda \rightarrow \lambda^{\prime}\right)=\frac{\exp \left\{-\beta\left[U\left(\mathbf{s}^{\prime N} ; \lambda^{\prime}\right)-\gamma A\left(\lambda^{\prime}\right)\right]\right\}}{\exp \left\{-\beta\left[U\left(\mathbf{s}^{N} ; \lambda\right)-\gamma A(\lambda)\right]\right\}}
$$

where $\gamma$ is the imposed surface tension. If we choose the particular value $\gamma=0$, then the explicit term depending on the area in equation 8 drops. The described scheme can be applied to impose any value of the surface tension. It is important to remark that this scheme assumes that the stress tensor is diagonal, which is true for fluid systems.

\section{Phase Behavior of Coarse-Grained Lipid Bilayers}

The phase behavior of different phosphocholines (PC's) has been determined experimentally (see [56] for a review). All PC's have a low temperature $L_{\beta^{\prime}}$ phase (see Fig. 4(a)). In this phase the bilayer is a gel: the chains of the phospholipids are ordered and show a tilt relative to the bilayer normal. At higher temperature the $L_{\alpha}$ phase is the stable phase. This phase is the liquid crystalline state of the bilayer in which the chains are disordered and tail overlap due to this thermal disorder is possible. This phase is physiologically the most relevant [57]. Under normal conditions the two monolayers of a bilayer contact each other at the terminal methyl group of their hydrophobic chains, while their hydrophilic headgroups are in contact with water. However, it is known experimentally that at low temperatures an interdigitated state, in which the terminal methyl groups of one monolayer interpenetrate the opposing layer, is also possible. This $L_{\beta I}$ phase does not spontaneously form in bilayers of symmetrical chain phospholipids, like dipalmitoylphosphatidylcholines (DPPC), [58] but can be induced by changes in the environment, like hydrostatic pressure or changes in the $\mathrm{pH}$ of the solution [59], or by incorporation, at the membrane interface of small amphiphilic molecules, like 


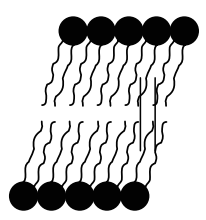

(a) $L_{\beta^{\prime}}$

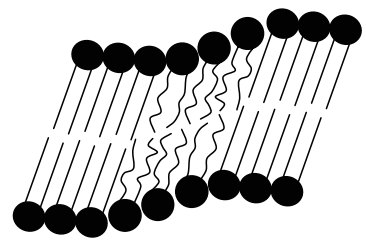

(b) $P_{\beta^{\prime}}$

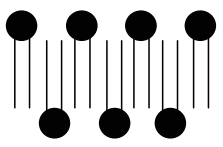

(d) $L_{\beta I}$

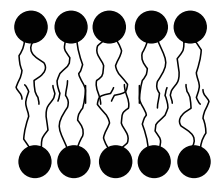

(c) $L_{\alpha}$

Fig. 4. Schematic drawings of the various bilayer phases. The characteristics of these phases are explained in the text. The filled circles represent the hydrophilic headgroup of a phospholipid and the lines represent the hydrophobic tails

alcohols [60-62], or anesthetics [63]. Interdigitation can also be induced by changes in the lipid structure, for example by introducing an ester-linkage in the headgroup of the phospholipids $[64,65]$.

An important question in the development of a mesoscopic model is how much chemical detail should be included in the model. Most likely this depend on the properties one would like to study. Here, we focus on the phase behavior and we illustrate how one can use mesoscopic models to obtain insights in how changes in the molecular structure or interactions of the lipid change its phase behavior. This insight can subsequently be used for the development of such a mesoscopic model.

\subsection{Single-Tail Lipid Bilayers}

Single-tail lipids are studied by several groups as models for phospholipid membranes [26] and it is an interesting question of such a simple amphiphilic molecule can describe the phase behavior of say DMPC. This question was addressed by Kranenburg et al. [27] using a model in which the lipid is represented by one head segment connected to a single tail with variable length as shown in Fig. 5. These authors have used the soft repulsion which are often used in DPD simulations. With this model the authors studied the effect of changes in the head-head interactions, caused, by, for example, adding salt to the system, on the phase diagram.

The computed phase diagram as a function of temperature and head-head interactions for the lipid $h t_{9}$ is shown in Fig. 6. If one would use this model to describe DMPC one would take a head-head repulsion of $a_{\mathrm{hh}}=35$. For this value of the head-head repulsion, an interdigitated gel phase, $L_{\beta I}$ is observed at low temperatures, while at $a_{\mathrm{hh}}=15$ the non-interdigitated $L_{\beta}$ phase is 


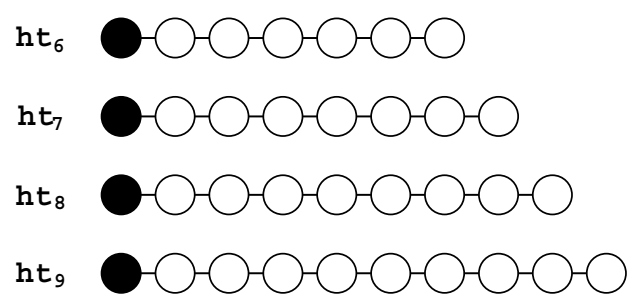

Fig. 5. Schematic drawing of the model lipids used by Kranenburg et al. [27]. The black particles represent the head beads and the white particles the tail beads. Two consecutive beads are connected by harmonic springs with spring constant and a harmonic bond bending potential between three consecutive beads is added with a bending constant $K_{\theta}=10$ and an equilibrium angle $\theta_{0}=180^{\circ}$. The soft-repulsion parameters used are $a_{\mathrm{ww}}=a_{\mathrm{tt}}=25, a_{\mathrm{wh}}=15$, and $a_{\mathrm{wt}}=80$

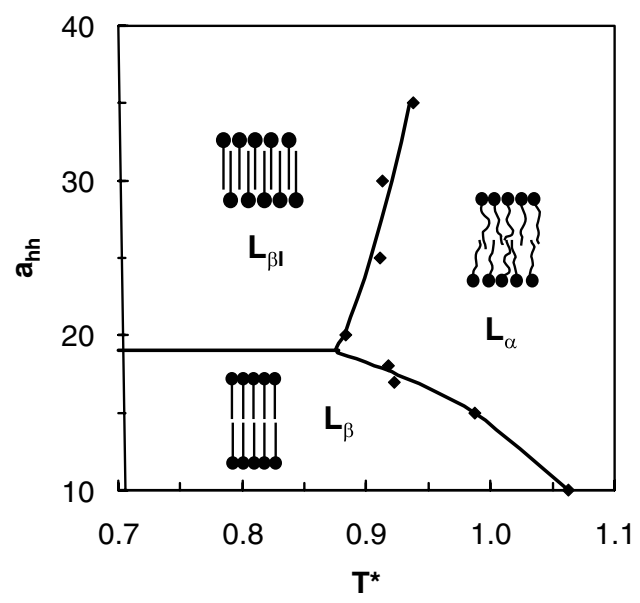

Fig. 6. Computed phase diagram of the lipid $h t_{9}$ as function of head-head repulsion parameter $a_{\mathrm{hh}}$ and reduced temperature $T^{*}$. At high values of the head-head repulsion parameters the interdigitated $L_{\beta I}$ phase is formed, while at low values the non-interdigitated $L_{\beta}$ phase is formed. Increasing temperature causes the melting of the bilayer to the $L_{\alpha}$ phase. These simulations involve a tensionless membrane of 200 lipids and the total number of particles was 3500 . The overall density of the system is $\rho=3$. The results are expressed in the usual reduced units, i.e. using $R_{c}$ as the unit of length and $k_{B} T_{o}=1$, with $T_{o}$ room temperature, as unit of the energy. $T^{*}$ is the temperature expressed in this unit

formed. The high temperature phase is the liquid or $L_{\alpha}$ phase. A snapshot of the different phases is given in Fig. 7.

\section{Characterization of the Bilayer Phases}

Before discussing this transition in detail, it is interesting to see what happens in the low and the high temperature phases. To characterize the ordering of 


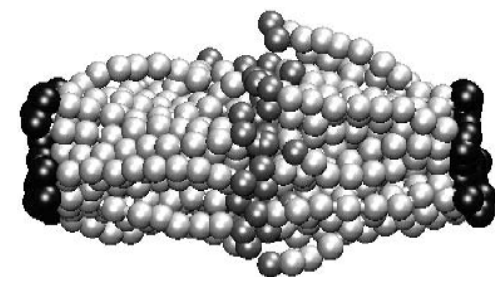

(a)

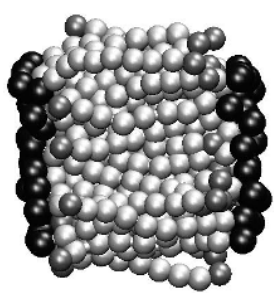

(b)

Fig. 7. Snapshots of the simulations of a bilayer consisting of the lipid $h t_{9}$ at $T^{*}=$ 0.85. (a) The non-interdigitated gel phase $L_{\beta}$ at $a_{\mathrm{hh}}=15$ and (b) the interdigitated gel phase $L_{\beta}$ at $a_{\mathrm{hh}}=15$. Black represents the hydrophilic headgroup and gray represents the hydrophobic tails

the lipids in the bilayer we use the order parameter $S_{\text {tail }}$, is a measure of the order in the tails. In Fig. $8 S_{\text {tail }}$ is plotted as a function of temperature. $S_{\text {tail }}$ has two regimes; below $T^{*}=0.95$ where $S_{\text {tail }}$ has values higher than 0.5 indicating that the chains are ordered along the bilayer normal, and above $T^{*}=0.95$ where the values of $S_{\text {tail }}$ decrease below 0.5 , showing an increase in the disorder of the chains.

The density profiles show that in the low temperature region, the two monolayers are interdigitated. The lipids stretch out in the direction normal to the bilayer, inducing interdigitation. This packing results in a larger average distance between the lipids headgroups in each monolayer and in a larger area. In this region an increase of temperature reduces the values of the order parameter, but along the chain the order persists. Thus interdigitation is still present, but is decreasing in depth, resulting in an increase of the bilayer thickness and a decrease of the area per lipid. Above the transition temperature, the chains loose the persisting order and are not interdigitated. Only the terminal tail beads overlap, due to thermal disorder. In this temperature region an increase in temperature increases the effective volume occupied by the molecules, but the extent of tail overlap does not depend significantly of temperature. As a result the area per molecule increases while the bilayer thickness decreases.

\section{Determination of the Phase Boundaries}

Three quantities have been used to distinguish among the different phases: the area per lipid $A_{\mathrm{L}}$, the extent of tail overlap $D_{\text {overlap}}$, and the ordering of the tails $S_{\text {tail }}$. Figure $8(\mathrm{a})$ shows the area per lipid $A_{\mathrm{L}}$, as function of temperature and head-head repulsion parameter. For repulsion parameters $a_{\mathrm{hh}} \leq 18$, the low temperature phase is the bilayer gel $L_{\beta}$ phase, while for repulsion parameters $a_{\mathrm{hh}}>18$, the low temperature phase is the interdigitated gel $L_{\beta I}$.

By increasing temperature all bilayers melt from an ordered into a disordered phase. For bilayers in the $L_{\beta}$ phase, the area per molecule and chain 


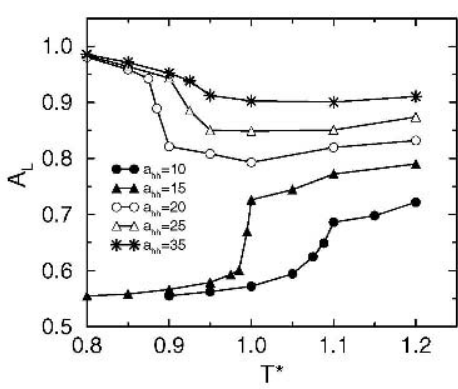

(a)

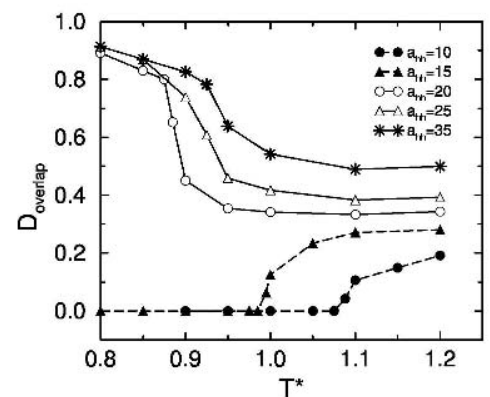

(b)

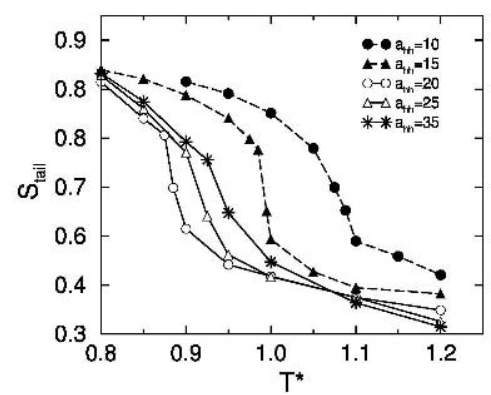

(c)

Fig. 8. (a) Area per lipid $A_{\mathrm{L}}$, (b) extent of chain overlap $D_{\text {overlap, }}$, and (c) tail order parameter $S_{\text {tail }}$ as function of reduced temperature $T^{*}$ for different repulsion parameters $a_{\mathrm{hh}}$. Dashed curves show a transition from the $L_{\beta}$ to the $L_{\alpha}$ phase, solid curves show the transition from the $L_{\beta I}$ to the $L_{\alpha}$ phase

overlap increase upon melting, while for bilayers in the $L_{\beta I}$ phase the area per molecule and chain overlap decrease.

The curves in Fig. 8(c) show that the transition from an ordered phase to a disordered one is very gradual. Much larger systems might be required to observe a sharp transition in these quasi two-dimensional systems. This gradual transition makes it difficult to determine the exact location of the phase boundaries and therefore we used the inflection point as our definition of the phase boundary. The temperature at which the chains get disordered is the same as the temperature of the inflection point in $A_{\mathrm{L}}$ and $D_{\text {overlap }}$. We define as the main transition temperature $T_{\mathrm{m}}$ the value of temperature at the inflection point of the shown curves. $T_{\mathrm{m}}$ is higher for bilayers in the $L_{\beta}$ phase than for bilayers in the $L_{\beta I}$ phase. This is in agreement with experimental results [59].

\section{Phase Behavior as a Function of Tail Length}

Besides investigating the effect of changing the head-head repulsion parameter, it is also interesting to vary the tail length of the lipid. A similar analysis, 


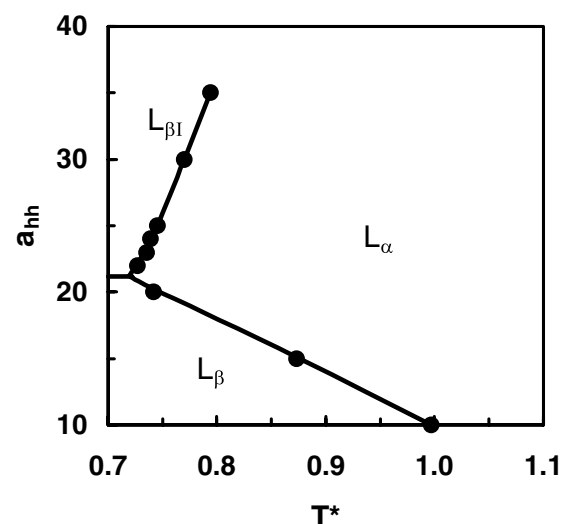

(a)

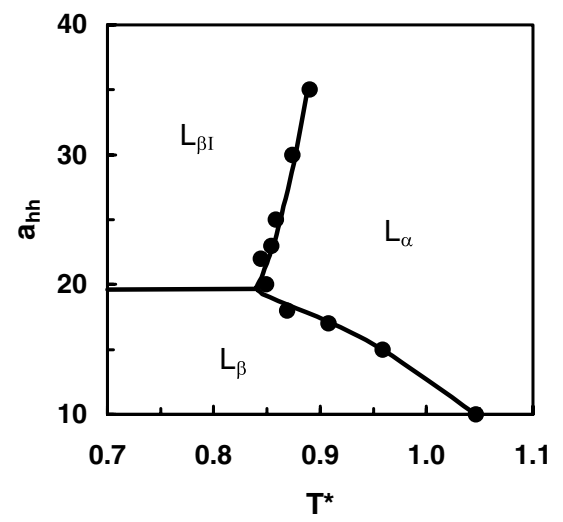

(c)

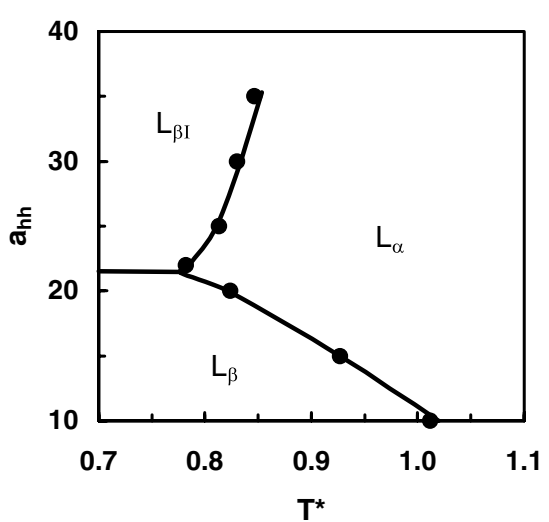

(b)

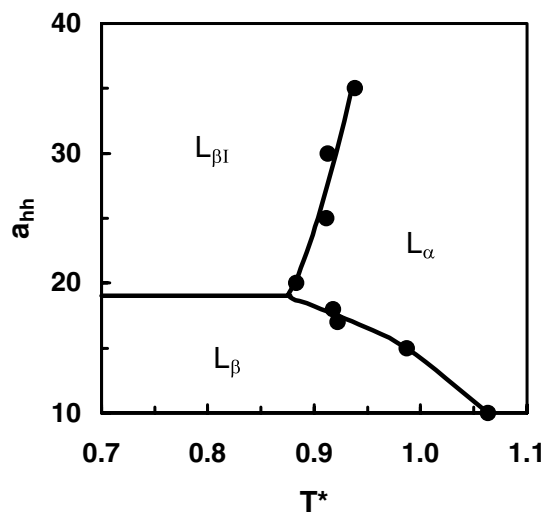

(d)

Fig. 9. Phase diagrams as a function of the head-head repulsion parameter $a_{\mathrm{hh}}$ and reduced temperature $T^{*}$ for lipids of different chain lengths: (a) $h t_{6}$ (b) $h t_{7}$ (c) $h t_{8}$, and $(\mathbf{d}) h t_{9}$

as was presented for the lipid $h t_{9}$, has been carried out for lipid types $h t_{6}, h t_{7}$, and $h t_{8}$ (see Fig. 9).

Depending on the repulsion parameter we obtain two gel phases $L_{\beta I}$ and $L_{\beta}$ for all tail lengths. For high head-head repulsion the system can gain energy by adding water particles in between the heads. As a result the distance between the head groups increases and the interdigitated phase is stabilized. For low values of $a_{\mathrm{hh}}$ the headgroups expel water and the stable phase is the non-interdigitated phase. In between we find $a_{\mathrm{hh}}^{*}$ for which the transition from $L_{\beta I}$ to $L_{\beta}$ occurs. The difference between the two phases is that in the $L_{\beta I}$ phase the tail ends are in direct contact with water, whereas in the $L_{\beta}$ phase the tail ends face each other. Therefore, the critical value $a_{\mathrm{hh}}^{*}$ to induce interdigitation is higher than the value of $a_{\mathrm{wh}}$. 
As we increase the tail length the gel phases are stabilized and the transition shifts to higher temperatures. The effect of increasing the head-head repulsion on the gel to liquid crystalline transition temperature is much more pronounced for the $L_{\beta} \rightarrow L_{\alpha}$ compared to $L_{\beta I} \rightarrow L_{\alpha}$. This can be understood from the fact that in the interdigitated phase the average distance between the heads is already much larger compared to the non-interdigitated phase, and a further increase in this distance does not have a dramatic effect on the stability of the gel phase.

For lipids $h t_{8}$ and $h t_{9}$ the $L_{\beta I}$ phase occurs at slightly lower repulsion parameters than for lipids $h t_{6}$ and $h t_{7}$. This is consistent with experimental results [66]. Since the interdigitated phase is more closely packed than the noninterdigitated phase, the van der Waals energy is greater. This energy gain is proportional to the number of carbon atoms in the phospholipid chain and thus interdigitation becomes energetically more favorable for longer chains. Also in our simulations we observe that the interdigitated phase is more compact and hence $a_{\mathrm{hh}}^{*}$ decreases slightly with increasing tail length.

It is interesting to compare these results with the experimental data. Misquitta and Caffrey in [67] systematically investigate the phase diagrams of monoacylglycerols, a single-tail lipid, and show a similar tail length dependence for the $\mathrm{L}_{\beta} \rightarrow \mathrm{L}_{\alpha}$ transition. Interestingly, as we will show later, for a similar model of a double-tail lipid we do not observe the spontaneous formation of an interdigitated phase. This corresponds to the experimental observation that for the most common double-tail lipids the interdigitated phase does not form spontaneously, but should be induced by the addition of, for example, alcohol [58].

\subsection{Double-Tail Lipid Bilayers}

In the previous section we have discussed the phase behavior of single-tail lipid bilayers. In this section we extend the model and investigate the phase behavior of a double-tail lipid with three head-beads and two tails of five beads each $\left(h_{3}\left(t_{5}\right)_{2}\right)$. The computed phase diagram is shown in Fig. 10. No interdigitation was found for the chosen values of the repulsion parameter between the headgroups. Also, an increase of $a_{\mathrm{hh}}$ up to 55 does not lead to any interdigitation (data not shown). This result is consistent with the experimentally observed structure of symmetric PC's bilayers, for which no spontaneous interdigitation is found. The snapshots in Fig. 11(c) shows a typical configuration of the system in the various phases.

At very low temperatures the system is in the $L_{\beta^{\prime}}$ gel phase, which is characterized by having ordered chains, hence a high value of the bilayer thickness and of the tail order parameter. While single-tail lipids are not tilted in the gel phase, for the double-tail lipid we observe that the lipid chains are tilted with respect to the bilayer normal. We find a tilt angle of $25^{\circ}$, which is slightly lower than the value of $\approx 32^{\circ}$ measured experimentally for DMPC 


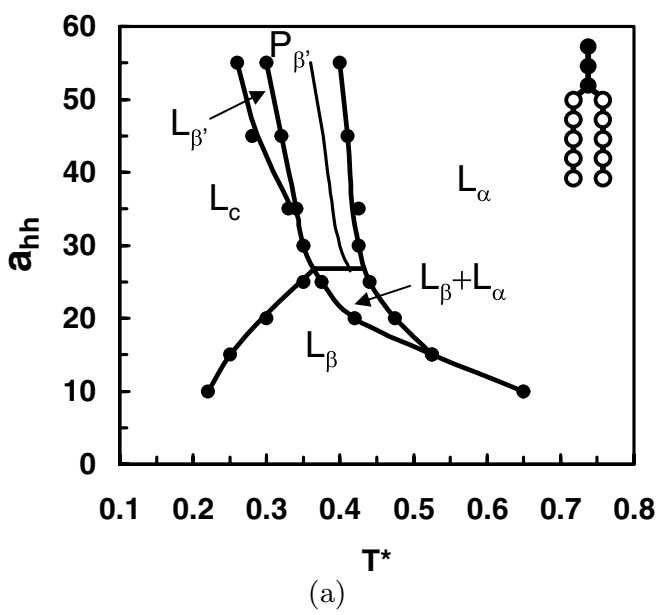

Fig. 10. Phase diagram of model lipid, $h_{3}\left(t_{5}\right)_{2}$, as a function of head-head repulsion and reduced temperature

lipid bilayers [68]. A typical configuration at this temperature can be see in the snapshot in Fig. 11(a).

Between the $L_{\alpha}$ and the $L_{\beta^{\prime}}$ phases, when the temperature is increased above $\mathrm{T}^{*}=0.35$, we observed a third phase. This phase, which disappears again as the temperature reaches the main-transition temperature, is characterized by having striated regions made of lipids in the gel-state intercalated by

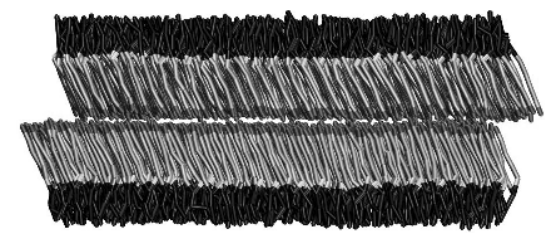

(a) $L_{\beta^{\prime}}$

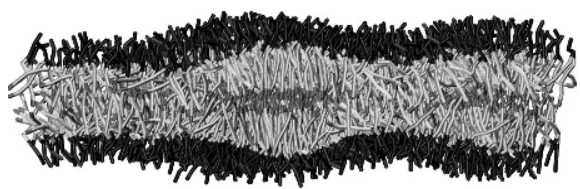

(b) $P_{\beta^{\prime}}$

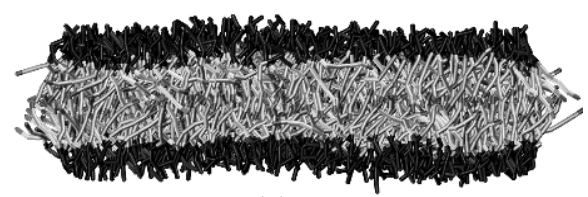

(c) $L_{\alpha}$

Fig. 11. Snapshots of typical configurations of the $h_{3}\left(t_{5}\right)_{2}$ bilayer simulated at reduced temperatures: (a) $\mathrm{T}^{*}<0.35$, corresponding to the gel phase, or $\mathrm{L}_{\beta^{\prime}}$; (b) $0.35 \leq \mathrm{T}^{*}<0.425$ corresponding to the ripple-like "striated" phase, or $\mathrm{P}_{\beta^{\prime}}$; and (c) $\mathrm{T}^{*}>0.425$ corresponding to the fluid phase, or $\mathrm{L}_{\alpha}$. The lipid headgroups are represented by black lines and the lipid tails by gray lines, with the terminal tail beads darker gray. The water is not shown 
Table 1. Values from simulation of the bilayer hydrophobic thickness, $D_{c}$, and area per lipid, $A_{L}$, at different temperatures, compared with experimental values. The error on the simulation data is $0.2 \AA$ for $D_{c}$ and $0.4 \AA^{2}$ for $A_{\mathrm{L}}$.

\begin{tabular}{cccccc}
\hline $\mathrm{T}\left[{ }^{\circ} \mathrm{C}\right]$ & Phase & \multicolumn{2}{c}{$D_{c}[\AA]$} & \multicolumn{2}{c}{$\mathrm{A}_{\mathrm{L}}\left[\AA^{2}\right]$} \\
& & $\operatorname{sim}$ & exper & $\operatorname{sim}$ & exper \\
\hline 10 & $L_{\beta^{\prime}}$ & 34.3 & $30.3^{\dagger}$ & 48.6 & $47.2^{\dagger}$ \\
30 & $L_{\alpha}$ & 26.3 & $25.6^{\ddagger}$ & 60.4 & $60.0^{\ddagger}$ \\
50 & $L_{\alpha}$ & 24.3 & $24.0^{\ddagger}$ & 64.4 & $65.4^{\ddagger}$ \\
65 & $L_{\alpha}$ & 23.6 & $23.4^{\ddagger}$ & 65.7 & $68.5^{\ddagger}$ \\
\hline
\end{tabular}

${ }^{\dagger}$ From [68]. The error for $D_{c}$ is $0.2 \AA$, and for $A_{\mathrm{L}} 0.5 \AA^{2}$.

$\ddagger$ From [70]. The error is not reported in the cited reference.

regions made of lipids in the fluid-state. This modulated structure can be seen in the snapshot in Fig. 11(b). This phase resembles the $P_{\beta^{\prime}}$, or ripple-phase. The ripple-phase occurs in phospholipid bilayers at the so-called pre-transition temperature, and is characterized by a rippling of the bilayer, with a wave length of the order of $150 \AA$ [69].

It is interesting to make a more quantitative comparison of our coursegrained model with a real phospholipid. The double-tail lipid $h_{3}\left(t_{5}\right)_{2}$ can be mapped onto DMPC, if a coarse-grained representation is used in which one DPD bead has a volume of $90 \AA^{3}{ }^{3}$ The unit of length is derived, from the volume of one DPD bead, and is equal to $R_{c}=6.4633 \AA$. To relate the temperatures in our model to real temperatures, we use the the main- and pre-transition temperatures for a pure DMPC phospholipid bilayer $\left(\approx 24{ }^{\circ} \mathrm{C}\right.$ and $\approx 14{ }^{\circ} \mathrm{C}[56]$ of the main- and pre-transition temperatures, respectively).

The values of the bilayer hydrophobic thickness and the area per lipid obtained from our simulations can now be compared with the corresponding experimental values for fully hydrated DMPC bilayers, as shown in Table 1 The values from simulations are in good quantitative agreement with the experimental data, although some deviations from the experimental values are observed for the area per lipid at high temperature $\left(65^{\circ} \mathrm{C}\right)$, and for the bilayer hydrophobic thickness in the gel phase $\left(10^{\circ} \mathrm{C}\right)$. The larger thickness, compared with the experimental value, found in our simulations in the gel phase, could be due to the smaller tilt angle shown by the coarse grained lipids compared to the DMPC lipids.

\section{Perturbations of the Membrane Structure}

In the previous section we have shown that one can obtain a very reasonable description of the phase behavior of a phospholipid membrane. Here we 
show that this model can be used to study the effect of adding alcohol or a transmembrane peptide on the structure of a membrane.

\subsection{Effect of Alcohol}

Small molecules such as alcohols or anesthesia can adsorb inside a membrane and this adsorption may induce changes in the structure of the membrane which can have an influence on the functioning of the membrane [58]. To study the effect of these molecules on the properties studies on various model membranes have been carried out by various groups $[66,71-76]$. These studies show that at low temperatures adding alcohol leads the formation of an interdigitated or $L_{\beta I}$ phase in stead of the commonly formed $L_{c}$ or $L_{\beta^{\prime}}$ (gel phases).

The structure of the interdigitated phase as been studied in detail and based on the available experimental data Adachi et al. [62] proposed the model shown in Fig. 12, in which the alcohol molecules fill the space and prevent the hydrophobic tails of the lipids to be exposed to water. This model nicely explains the experimentally observed density profiles and provides a simple molecular explanation why the alcohol molecules stabilize the interdigitated phase. This model also suggests that the optimal alcohol to lipid ratio in the membrane is $2: 1$. Therefore, measuring this ratio in a membrane would be an important conformation of this model. However, experimentally it is very difficult to measure the concentration of alcohol in the membrane directly. Most studies use theoretical models to relate the alcohol concentration in the water phase to the alcohol concentration in the membrane. Since the validity of these models has not been tested, it is not yet clear whether the 2:1 ratio can indeed be confirmed experimentally.

The model of the alcohols consists of one hydrophilic head bead an a tail that varies in length from one to three hydrophobic beads. In the coarsegraining procedure the alcohols methanol through pentanol correspond with the coarse-grained models $h t$ and $h t_{2}$ and hexanol and heptanol correspond

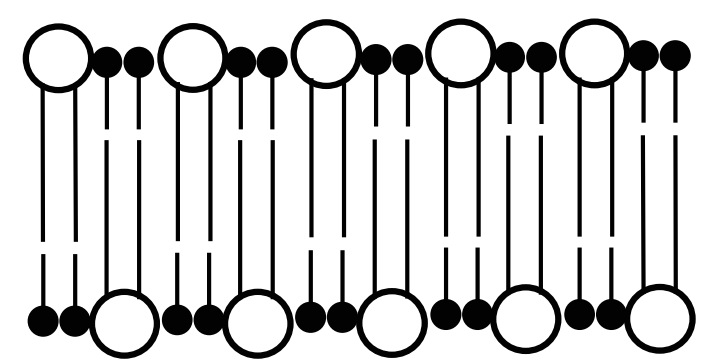

Fig. 12. Proposed model of the interdigitated phase by alcohols [62], in which every tail end of a phospholipid is facing the tail end of an alcohol. Black molecules represent the phospholipids and gray molecules the alcohols 


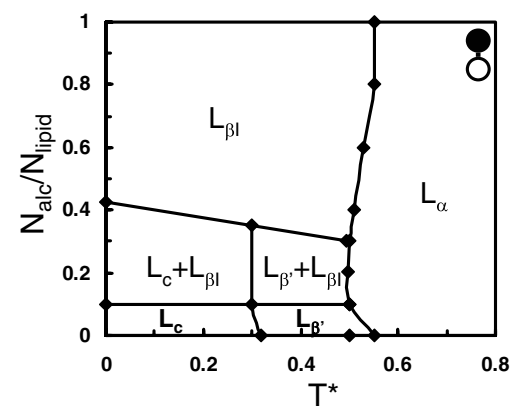

(a)

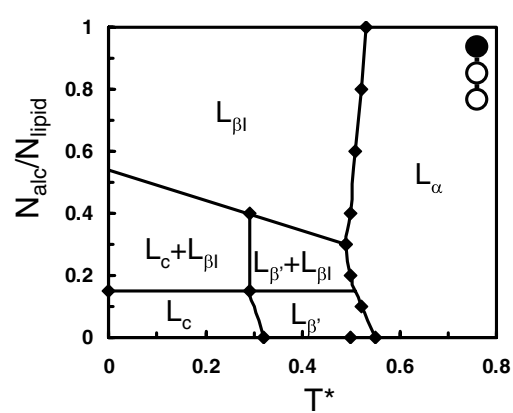

(b)

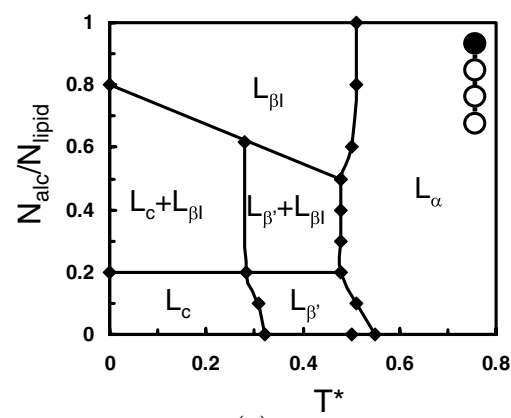

(c)

Fig. 13. Phase diagrams of the lipid $h_{3}\left(t_{7}\right)_{2}$ with the model alcohols $h t(\mathbf{a}), h t_{2}$ (b), and $h t_{3}(\mathbf{c})$

with the model $h t_{3}$. The bond-bending potential with $k_{\theta}=6$ and $\theta=180^{\circ}$ was also applied between three consecutive beads of the these alcohols.

Figure 13 shows the effect of alcohols with different tail lengths on the phase behavior of a membrane. At zero alcohol concentration we nicely recover the experimentally observed phases. At low temperatures we observe the $L_{c}$ phase, in which the tails are order and tilted. If we increase the temperature the tails lose their order in the $L_{\beta^{\prime}}$ phase, and at even higher temperatures the tails also loose their tilt in the fluid or $L_{\alpha}$ phase.

At low concentrations of alcohol, the molecules are homogeneously distributed in the $L_{\beta^{\prime}}$ or $L_{c}$ phase. At high alcohol concentrations we find the $L_{\beta I}$, in which the lipid tails do not have a tilt with respect to the bilayer normal. The tails of the lipids of one monolayer are fully interpenetrated into the opposing layer and the tail ends are facing the tail end of the alcohol. In between these two extremes we find that there is coexistence between the interdigitated and non-interdigitated phase. The alcohols are inhomogeneously distributed in the bilayer and mainly located in the interdigitated part of the bilayer.

At lower temperatures the concentration of alcohol required to obtain the fully interdigitated phase increases, which is in agreement with the experimental observations of Nambi et al. [72]. Our simulations also report the so-called 
"biphasic effect" on the transition from the low temperature gel phase to the high temperature fluid phase [71]; at low concentrations of alcohol, the main transition temperature shifts to a lower temperature, while at high concentrations this transition temperature shifts to a higher temperature compared to a pure lipid bilayer. Figure 13 shows that the increase or decrease in temperature are directly related to the effect of the alcohol on the stability of the low temperature phases [66]. The $L_{\beta^{\prime}}$, phase is destabilized by alcohol, while the $L_{\beta I}$ can easily incorporate alcohol molecules.

The phase diagrams also show the effects of changes of the length of the alcohol. We observe little influence on the $L_{\beta I} \rightarrow L_{\alpha}$ transition. However, we do observe a difference in the stability of the $L_{\beta I}$ phase as a function of the concentration of alcohol; the shorter the alcohol, the more stable $L_{\beta I}$ phase.

An important issue is the concentration of alcohol in the bilayer. The critical bulk concentration at which interdigitation is complete decreases with increasing length of the alcohol $[71,75,77]$. Various theories have been used to relate the bulk concentration of alcohol to the concentration in the lipid. At present there is little consensus in the literature whether this procedure yields a reliable estimate of the alcohol concentration in the bilayer [78-82]. In our simulations we can compute the number of alcohols in the bilayer directly. Comparison of the phase diagrams (Fig. 13) for three different alcohols shows that if we increase the length of the alcohol more alcohol molecules are needed to destabilize the $L_{c}$ and $L_{\beta}$ phases. The $L_{\beta I}$ requires a higher concentration of the longer chain alcohols to be stable, which agrees nicely with experimental results [77].

Adachi et al. [62] proposed a model of the structure of the bilayer in which the terminal methyl group of the alcohol faces a terminal methyl group of a lipid chain (see Fig. 12). The assumption is based on the experimental observation that the membrane thickness increases by about $0.08 \mathrm{~nm}$ per one methylene unit in either the alcohol molecules or the phospholipids $[62,83]$. This distance of $0.1 \mathrm{~nm}$ is the length of one $\mathrm{CH}_{2}$-unit in the stretched chain of an alkane [84]. Furthermore, Adachi et al. [62] show that two alcohol molecules can occupy a volume surrounded by the PC head groups of one layer. From this it follows that the number of alcohol molecules should be twice as high as the number of lipids in the bilayer independent of the length of the alcohol. This conclusion clearly differs with the results of our simulations which do indicate that the stability of the interdigitated phase depends on the length of the alcohol.

We observe that the fully interdigitated phase occurs at much lower number of alcohols than twice the number of lipids in the lipid bilayer. This can be explained by taking into account an energy balance between the noninterdigitated and interdigitated phase. By the incorporation of alcohols at the membrane interface, voids are created in the hydrophobic core, which are energetically

unfavorable. The more alcohol, the higher the energy of the membrane. In the interdigitated phase the tail ends of the lipid are in contact with the 
interfacial water. Adding alcohol reduces these energetically unfavorable interactions and hence increasing the alcohol concentration decreases the energy of the membrane. Clearly at the $2: 1$ ratio, the energy of the interdigitated phase will be lowest, but at much lower alcohol concentrations the energy can already be lower compared to the $L_{\beta^{\prime}}$ phase. To use this model quantitatively, one would need to take entropy effects into account as well, but this model does rationalize why we observe in our simulations already an interdigitated phase at much lower alcohol concentrations.

\subsection{Bilayers with Transmembrane Proteins}

The hydrophobic matching between the lipid bilayer hydrophobic thickness and the hydrophobic length of integral membrane proteins has been proposed as a generic physical principle on which the lipid-protein interaction in biomembranes is based [14,20,85-88]. The energy cost of exposing polar moieties, from either hydrocarbon chains or protein residues, is so high that the hydrophobic part of the lipid bilayer should match the hydrophobic domain of membrane proteins. The results from a number of investigations have indeed pointed out the relevance of the hydrophobic matching in relation to the lipid-protein interactions, hence to membrane organization and biological function.

In this work we study the perturbation caused by a transmembrane peptide on the surrounding lipids, its possible dependence on hydrophobic mismatch, protein size, and on temperature. We have investigated whether and to which extent - due to hydrophobic mismatch and via the cooperative nature of the system - a protein may prefer to tilt (with respect to the normal to the bilayer plane), rather than to induce a bilayer deformation without (or even with) tilting.

The systems that we have simulated are made of model lipids having three headgroup beads and two tails of five beads each; this corresponds to the case of acyl chains with fourteen carbon atoms, namely to a model for a dimyristoylphosphatidylcholine (DMPC) phospholipid, as illustrated in in Fig. 14(a).

Within the model formulation, a peptide is considered as a rod-like object, with no appreciable internal flexibility, and characterized by a hydrophobic length $d_{\mathrm{P}}$. The model for the transmembrane peptide is built by connecting $n_{t_{P}}$ hydrophobic beads into a chain, to which ends $n_{h}$ headgroup-like beads are attached. $N_{\mathrm{P}}$ of these amphiphatic chains are linked together into a bundle. In each model protein, all the $N_{\mathrm{P}}$ chains are linked to the neighboring ones by springs, to form a relatively rigid body. We have considered three typical model-protein sizes, two of them referring to a "skinny" peptide-like molecule, and the third type to a "fat" protein. These model proteins consist of $N_{\mathrm{P}}=4$, 7 , or 43 chains linked together in a bundle. The bundle of $N_{\mathrm{P}}=7$ chains is formed by a central chain surrounded by a single layer of six other chains. The $N_{\mathrm{P}}=43$ bundle is made of three layers arranged concentrically around a central chain, and containing each six, twelve, and twenty four amphiphatic 


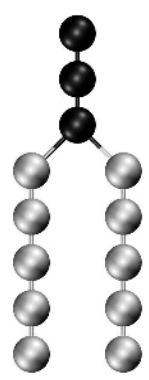

(a)

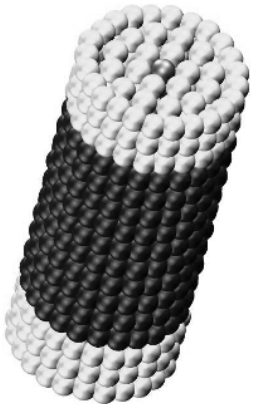

(b)

Fig. 14. Schematic representation of a model-lipid (a), and a model protein $\left(N_{\mathrm{P}}=\right.$ 43 and $\left.\tilde{d}_{\mathrm{P}}=41 \AA\right)(\mathbf{b})$

chains, respectively. Figure 14(b) shows a cartoon of a model protein of size $N_{\mathrm{P}}=43$.

One of the quantities that can be measured experimentally is the tilt of the peptide. The peptide tilt angle with respect to the bilayer normal as function of $\Delta d$ and peptide size $N_{\mathrm{P}}$ is shown in Fig. 15. The snapshots on the right show typical configurations of the system, for a fixed value of the peptide hydrophobic length, for the three peptide sizes, $N_{\mathrm{P}}=4,7$, and 43 , and for the largest (positive) value of mismatch, $\Delta d=26 \AA$, at the considered temperature, $\mathrm{T}^{*}=0.7$. For $\Delta d<0$ the tilt angle is very small, and is within the statistical tilt-fluctuations to which the peptide is subject in the bilayer; as the peptide hydrophobic length increases (and the mismatch becomes positive), the peptide undergoes a significant tilting. Also, for equal values of hydrophobic mismatch, the "thinner" peptide $\left(N_{\mathrm{P}}=4\right)$ is much more tilted than the "fatter" one $\left(N_{\mathrm{P}}=43\right)$. These results, combined with the one discussed above, suggest that in the case of peptides with small surface area, the main mechanism to compensate for a large hydrophobic mismatch is the tilt, while in the case of peptides with a large surface area, that cannot accommodate a too large tilt, the mismatch is mainly compensated for by an increase of the bilayer thickness around the peptide, as is clearly illustrated by the snapshot in Fig. $15\left(N_{\mathrm{P}}=43\right)$.

The occurrence of peptide/protein tilting has also been confirmed experimentally. In fact, the results from very recent experimental investigations by solid state NMR spectroscopy [89] show that $\alpha$-helical model peptides - of fixed hydrophobic length and with a hydrophobic leucine-alanine core, and tryptophan flanked ends - experience tilt when embedded in phospholipid bilayers of varying hydrophobic thickness (such that $d_{\mathrm{P}} \geq d_{\mathrm{L}}^{o}$, i.e. $\Delta d>0$ ). It was found that the tilt angle increases by systematically increasing hydrophobic mismatch; however, the tilt dependence on hydrophobic mismatch was not as pronounced as one would have expected, given the degree of mismatch. This result brought the authors to conclude that the tilt of these peptides is ener- 

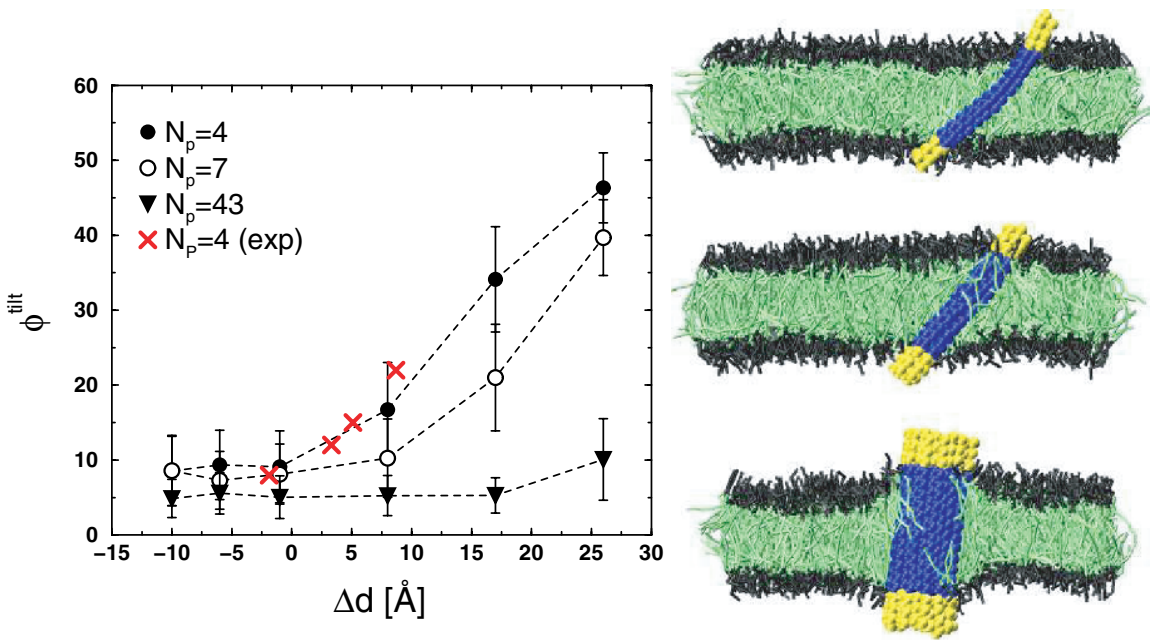

Fig. 15. peptide tilt-angle $\phi^{\text {tilt }}$ as function of mismatch, $\Delta d$. The data refer to a reduced temperature of $\mathrm{T}^{*}=0.7$ and to the three considered peptide sizes $N_{\mathrm{P}}=4$, 7 and 43. The dashed lines are only meant to be a guideline for the eye and the crosses are experimental data. Typical configurations of the systems resulting from the simulations are shown on the right. Starting from the top, the snapshots refer to peptides sizes $N_{\mathrm{P}}=4,7$ and 43 . In the three cases the peptide hydrophobic length is $\tilde{d}_{\mathrm{P}}=50 \AA$, hence the hydrophobic mismatch is $\Delta d=26 \AA$

getically unfavorable, and to suggest that the (anchoring) effects by specific residues such as tryptophans are more dominant than mismatch effect. A large tilt is instead experienced by the M13 coat peptide peptide when embedded in phospholipid bilayer of varying hydrophobic thickness [90]. For values of mismatch of the same order of the one experienced by the synthetic peptides just mentioned [89], the degree of tilting experienced by M13 peptide is much higher. Our simulation data indicate a dependence of the peptide-tilt angle on mismatch in agreement with the experimental data just discussed. Incidentally, the results from our simulations suggest that, when a skinny peptide $\left(N_{\mathrm{p}}=4\right)$ is subjected to a large positive mismatch $\left(d_{\mathrm{P}}>d_{\mathrm{L}}^{\mathrm{o}}\right)$, it might bend besides to experience a tilt - as can be seen by looking at the snapshot shown on the top-right of Fig. 15. Also, as soon as the positive mismatch decreases, the bending disappears, although the peptide still tends to remain tilted.

\section{Concluding Remarks}

In this contribution we have shown that a simple, mesoscopic, representation of a phospholipid can give a surprisingly realistic description of the phase behavior of biological membranes. The simulations showed that different stable phases are obtained for a wide range of temperatures. We characterized the low 
temperature phase as a gel phase, and we reproduced the main order/disorder phase transition from a gel to a liquid crystalline phase.

We also demonstrate that this model can be used to study the effect of alcohol on the structure of the membrane. Our model nicely reproduces the experimental phase diagram and the alcohol length dependence of the thickness of the interdigitated phase. Our simulations show that the interdigitated phase is stable at much lower alcohol concentrations in the membrane than suggested in the literature. This points at an alternative interpretation of the structure of the interdigitated phase.

This model has also been extended to lipid bilayers containing just one lipid species and an embedded protein. For which we have investigated the effect due to mismatch and protein size on the perturbation induced by the protein on the surrounding lipid bilayer.

\section{References}

1. C. Tanford (1978) The hydrophobic effect and the organization of living matter. Science 200, pp. 1012-1018

2. C. Tanford (1980) Hydrophobic Effect: Formation of Micelles and Biological Membranes. Wiley, New York

3. E. Sackmann (1995) Biological membranes architecture and function. In Structure and Dynamics of Membranes (R. Lipowsky and E. Sackmann, eds.), (Amsterdam), Elsevier pp. 1-65

4. S. König and E. Sackmann (1996) Molecular and collective dynamics of lipid bilayers. Current Opinion in Colloid and Interface Sci. 1, pp. 78-82

5. D. P. Tieleman, S. J. Marrink, and H. J. C. Berendsen (1997) A computer perspective of membranes: molecular dynamics studies of lipid bilayer systems. Biochim. Biophys. Acta 1331, pp. 235-270

6. S. J. Marrink and D. P. Tieleman (2001) Molecular dynamics simulation of a lipid diamond cubic phase. J. Am. Chem. Soc. 123, pp. 12383-12391

7. L. Shen, D. Bassolino, and T. Stouch (1997) Transmembrane helix structure, dynamics, and interactions: Multi-nanosecond molecular dynamics simulations. Biophys. J. 73, pp. 3-20

8. K. Belohorcová, J. A. Davis, T. B. Woolf, and B. Roux (1997) Structure and dynamics of an amphiphilic peptide in a bilayer: a molecular dynamics study. Biophys. J. 73, pp. 3039-3055

9. K. Belohorcová, J. Qian, and J. A. Davis (2000) Molecular dynamics and ${ }^{2} \mathrm{~h}-$ nmr study of the influence of an amphiphilic peptide on membrane order and dynamics. Biophys. J. 79, pp. 3201-3016

10. M. Ø. Jensen, E. Tajkhorshid, and K. Schulten (2001) The mechanism of glycerol conduction in aquaglyceroporins. Structure 9, pp. 1083-1093

11. M. Ø. Jensen and O. G. Mouritsen (2004) Lipids do influence protein function - the hydrophobic matching hypothesis revised. Biochim. Biophys. Acta: Biomembranes vol. in press, pp. 205-226

12. T. Stouch and D. Bassolino (1996) Movement of small molecules in lipid bilayers: Molecular dynamics simulation studies. In Biological Membranes (J. Mertz and R. B. eds.), (Boston), pp. 255-277; Birkhauser 
13. O. G. Mouritsen, M. M. Sperotto, J. Risbo, Z. Z. Zhang, and M. J. Zuckermann (1996) Computational approach to lipid-protein interactions in membranes. Adv. Comp. Biol. 2, pp. 15-64

14. T. Gil, J. H. Ipsen, O. G. Mouritsen, M. C. Sabra, M. M. Sperotto, and M. J. Zuckermann (1998) Theoretical analysis of protein organization in lipid membranes. Biochim. Biophys. Acta 1376, pp. 245-266

15. T. Gil and J. H. Ipsen (1997) Capillary condensation between disks in two dimensions. Phys. Rev. E 55, pp. 1713-1721

16. S. May (2000) Theories on structural perturbations of lipid bilayers. Curr. Opin. Colloid Interface Sci. 5, pp. 244-249

17. D. R. Fattal and A. Ben-Shaul (1993) A molecular model for lipid-protein interactions in membranes: the role of hydrophobic mismatch. Biophys. J. 65, pp. 1795-1809

18. C. Nielsen, M. Goulian, and O. S. Andersen (1998) Energetics of inclusioninduced bilayer deformations. Biophys. J. 74, pp. 1966-1983

19. A. G. Lee (2003) Lipid-protein interactions in biological membranes: a structural perspective. Biochim. Biophys. Acta 1612, pp. 1-40

20. J. A. Killian (1998) Hydrophobic mismatch between proteins and lipids in membranes. Biochim. Biophys. Acta 1376, pp. 401-416

21. R. Goetz and R. Lipowsky (1998) Computer simulations of bilayer membranes: Selfassembly and interfacial tension. J. Chem. Phys. 108, pp. 7397-7409

22. J. C. Shelley, M. Y. Shelley, R. C. Reeder, S. Bandyopadhyay, P. B. Moore, and M. L. Klein (2001) Simulations of phospholipids using a coarse grain model. $J$. Phys. Chem. B 105, pp. 9785-9792

23. J. C. Shelley, M. Y. Shelley, R. C. Reeder, S. Bandyopadhyay, and M. L. Klein (2001) A coarse grain model for phospholipid simulations. J. Phys. Chem. B 105, pp. 4464-4470

24. R. D. Groot and K. L. Rabone (2001) Mesoscopic simulation of cell membrane damage, morphology change and rupture by nonionic surfactants. Biophys. J. 81, pp. $725-736$

25. S. J. Marrink, A. H. de Vries, and A. E. Mark (2004) Coarse grained model for semiquantitative lipid simulations. J. Phys. Chem. B 108, pp. 750-760

26. M. Venturoli and B. Smit (1999) Simulating the self-assembly of model membranes. Phys. Chem. Comm. 2, part. no. 10

27. M. Kranenburg, M. Venturoli, and B. Smit (2003) Molecular simulations of mesoscopic bilayer phases. Phys. Rev. E 67, part. no. 060901

28. M. Kranenburg, M. Venturoli, and B. Smit (2003) Phase behavior and induced interdigitation in bilayers studied with dissipative particle dynamics. J. Phys. Chem. B 107, pp. 11491-11501

29. M. Kranenburg and B. Smit (2004) Simulating the effect of alcohol on the structure of a membrane. FEBS Lett. 568, no. 1-3, pp. 15-18, FEBS LETT

30. M. Kranenburg, J.-P. Nicolas, and B. Smit (2004) Comparison of mesoscopic phospholipid-water models. Phys. Chem. Chem. Phys. 6, no. 16, pp. 4142-4151

31. M. Kranenburg, M. Vlaar, and B. Smit (2004) Simulating induced interdigitation in membranes. Biophys. J. 87, pp. 1596-1605

32. M. Kranenburg, C. Laforge, and B. Smit (2004) Mesoscopic simulations of phase transitions in lipid bilayers. Phys. Chem. Chem. Phys. 6, pp. 4531-4534

33. M. Venturoli, B. Smit, and M. M. Sperotto (2005) Simulation studies of proteininduced bilayer deformations, and lipid-induced protein tilting, on a mesoscopic model for lipid bilayers with embedded proteins. Biophys. J. 88, pp. 1778-1798 
34. M. Kranenburg and B. Smit (2005) Phase behavior of model lipid bilayers. J. Phys. Chem. B 109, pp. 6553-6563

35. P. J. Hoogerbrugge and J. M. V. A. Koelman (1992) Simulating microscopic hydrodynamics phenomena with dissipative particle dynamics. Europhys. Lett. 19, pp. $155-160$

36. P. Español (2002) Dissipative particle dynamics revisited. SIMU, Challenges in Molecular Simulations 4, pp. 59-77

37. P. B. Warren (1998) Dissipative particle dynamics. Curr. Opinion Coll. Interface Sci. 3, pp. 620-624

38. J. C. Shillcock and R. Lipowsky (2002) Equilibrium structure and alteral stress distribution of amphiphilic bilayers from dissipative particle dynamics simulations. J. Chem. Phys. 117, pp. 5048-5061

39. G. Ayton and G. A. Voth (2002) Bridging microscopic and mesoscopic simulations of lipid bilayers. Biophys. J. 83, pp. 3357-3370

40. R. D. Groot and P. B. Warren (1997) Dissipative particle dynamics: bridging the gap between atomistic and mesoscopic simulation. J. Chem. Phys. 107, pp. $4423-4435$

41. P. Español and P. Warren (1995) Statistical mechanics of dissipative particle dynamics. Europhys. Lett. 30, pp. 191-196

42. S. M. Willemsen, T. J. H. Vlugt, H. C. J. Hoefsloot, and B. Smit (1998) Combining dissipative particle dynamics and Monte Carlo techniques. J. Comp. Phys. 147, pp. $507-517$

43. J. S. Rowlinson and B. Widom (1982) Molecular Theory of Capillarity. Oxford: Clarendon Press

44. F. Jähnig (1996) What is the surface tension of a lipid membrane? Biophys. J. 71, pp. 1348-1349.

45. R. Kwok and E. Evans (1981) Thermoelasticity of large lecithin bilayer vesicles. Biophys. J. 35, pp. 637-652

46. S.-W. Chiu, M. Clark, S. Subramaniam, H. L. Scott, and E. Jakobsson (1995) Incorporation of surface tension into molecular dynamics simulation of an interface: a fluid phase lipid bilayer membrane. Biophys. J. 69, pp. 1230-1245

47. R. Zhang, W. Sun, S. Traistram-Nagle, R. L. Headrick, R. M. Suter, and J. F. Nagle (1995) Critical fluctuations in membranes. Phys. Rev. Lett. 74, pp. 28322835

48. S. E. Feller and R. W. Pastor (1996) On simulating lipid bilayers with an applied surface tension: periodic boundary conditions and undulations. Biophys. J. 71, pp. $1350-1355$

49. S. E. Feller and R. W. Pastor (1999) Constant surface tension simulations of lipid bilayers: the sensitivity of surface areas and compressibilities. J. Chem. Phys. 111, pp. 1281-1287

50. S. J. Marrink, E. Lindahl, O. Edholm, and A. E. Mark (2001) Simulation of the spontaneous aggregation of phospholipids into bilayers. J. Am. Chem. Soc. 123, pp. 8638-8639

51. S. E. Feller, Y. Zhang, and R. W. Pastor (1995) Computer simulation of liquid/liquid interfaces. ii. surface tension-area dependence of a bilayer and monolayer. J. Chem. Phys. 103, pp. 10267-10276

52. D. P. Tieleman and H. J. C. Berendsen (1996) Molecular dynamics simulations of a fully hydrated dipalmitoylphosphatidylcholine bilayer with different macroscopic boundary conditions and parameters. J. Chem. Phys. 105, pp. 4871-4880 
53. S.-W. Chiu, E. Jakobsson, S. Subramaniam, and H. L. Scott (1999) Combined Monte Carlo and molecular dynamics simulation of fully hydrated dioleyl and palmitoyloleyl phosphatidylcholine lipid bilayers. Biophys. J. 77, pp. 2462-2469

54. R. M. Venable, B. R. Brooks, and R. W. Pastor (2000) Molecular dynamics simulations of gel $\left(l_{\beta i}\right)$ phase lipid bilayers in constant pressure and constant surface area ensemble. J. Chem. Phys. 112, pp. 4822-4832

55. F. Sun (2002) Constant normal pressure, constant surface tension, and constant temperature molecular dynamics simulation of hydrated 1,2dilignoceroylphosphatidylcholine monolayer. Biophys. J. 82, pp. 2511-2519

56. R. Koynova and M. Caffrey (1998) Phases and phase transitions of the phosphatidylcholines. Biochim. Biophys. Acta 1376, pp. 91-145

57. J. Katsaras and T. Gutberlet (2001) Lipid Bilayers, Structure and Interactions Berlin: Springer

58. J. L. Slater and C. H. Huang (1988) Interdigitated bilayer membranes. Prog. Lipid. Res. 27, pp. 325-359

59. S. Furuike, V. G. Levadny, S. J. Li, and M. Yamazaki (1999) Low pH induces an interdigitated gel to bilayer gel phase transition in dihexadecylphosphatidylcholine membrane. Biophys. J. 77, pp. 2015-2023

60. T. J. McIntosh, R. V. McDaniel, and S. A. Simon (1983) Induction of an interdigitated gel phase in fully hydrated phosphatidylcholine bilayers. Biochim. Biophys. Acta 731, pp. 109-114

61. T. J. McIntosh, H. Lin, S. Li, and C.-H. Huang (2001) The effect of ethanol on the phase transition temperature and the phase structure of monounsaturated phosphatidylcholines. Biochim. Biophys. Acta 1510, pp. 219-230

62. T. Adachi, H. Takahashi, K. Ohki, and I. Hatta (1995) Interdigitated structure of phospholipid-alcohol systems studied by X-ray diffraction. Biophys. J. 68, pp. $1850-1855$

63. T. Hata, H. Matsuki, and S. Kaneshina (2000) Effect of local anesthetics on the bilayer membrane of dipalmitoylphosphatidylcholine: interdigitation of lipid bilayer and vesicle-micelle transition. Biophys. Chem. 87, pp. 25-36

64. R. N. A. H. Lewis, I. Winter, M. Kriechbaum, K. Lohner, and R. N. McElhaney (2001) Studies of the structure and organization of cationic lipid bilayer membranes: Calorimetric, spectroscopic, and X-ray diffraction studies of linear saturated P-O-ethyl phosphatidylcholines. Biophys. J. 80, pp. 1329-1342

65. I. Winter, G. Pabst, M. Rappolt, and K. Lohner (2001) Refined structure of 1,2-diacyl-P-O-ethylphosphatidylcholine bilayer membranes. Chem. Phys. Lip. 112, pp. 137-150

66. S. A. Simon and T. J. McIntosh (1984) Interdigitated hydrocarbon chain packing causes the biphasic transition behavior in lipid/alcohol suspensions. Biochim. Biophys. Acta 773, pp. 169-172

67. Y. Misquitta and M. Caffrey (2001) Rational design of lipid molecular structure: a case study involving the c19:1c10 monoacylglycerol. Biophys. J. 81, pp. 10471058

68. S. Tristram-Nagle, Y. Liu, J. Legleiter, and J. F. Nagle (2002) Structure of gel phase dmpc determined by X-ray diffraction. Biophys. J. 83, pp. 3324-3335

69. R. S. Cantor (1998) The lateral pressure profiles in membranes: a physical mechanism of general anesthesia. Toxicology Lett. 101, pp. 451-458

70. J. G. Petrov, E. E. Polymeropoulos, and Möhwald (2000) Langmuir monolayers with fluorinated groups in the hydrophilic head. 1. comparison of trifluoroethyl 
behenate and ethyl behenate monolayers: Molecular models, mechanical properties, stability. Langmuir 16, pp. 6411-7420

71. E. S. Rowe (1983) Lipid chain length and temperature dependence of ethanolphosphatidylcholine interactions. Biochemistry 22, pp. 3299-3305

72. P. Nambi, E. S. Rowe, and T. J. McIntosh (1988) Studies of the ethanolinduced interdigitated gel phase in phosphatidylcholines using the fluorophore 1,6-diphenyl-1,3,5-hexatriene. Biochemistry 27, pp. 9175-9182

73. K. Ohki, K. Tamura, and I. Hatta (1990) Ethanol induces interdigitated gel phase $\left(l_{\beta i}\right)$ between lamellar gel phase $\left(l_{\beta^{\prime}}\right)$ and ripple phase $\left(p_{\beta^{\prime}}\right)$ in phosphatidylcholine membranes: a scanning density meter study. Biochim. Biophys. Acta 1028, pp. 215-222

74. U. Vierl, L. Löbbecke, N. Nagel, and G. Cevc (1994) Solute effects on the colloidal and phase behavior of lipid bilayer membranes: ethanoldipalmitoylphosphatidylcholine mixtures. Biophys. J. 67, pp. 1067-1079

75. L. Löbbecke and G. Cevc (1995) Effects of short-chain alcohols on the phase behavior and interdigitation of phosphatidylcholine bilayer membranes. Biochim. Biophys. Acta 1237, pp. 59-69

76. J. A. Veiro, P. Nambi, L. L. Herold, and E. S. Rowe (1987) Effect of $n$-alcohols and glycerol on the pretransition of dipalmitoylphosphatidylcholine. Biochim. Biophys. Acta 900, pp. 230-238

77. E. S. Rowe and J. M. Campion (1994) Alcohol induction of interdigitation in distearoylphosphatidylcholine: fluorescence studies of alcohol chain length requirements. Biophys. J. 67, pp. 1888-1895

78. S. A. Simon, W. L. Stone, and P. Busto-Latorre (1977) A thermodynamic study of the partition of n-hexane into phosphatidylcholine and phosphatidylcholinecholesterol bilayers. Biochim. Biophys. Acta 468, pp. 378-388

79. C. Trandum and P. Westh (2000) Alcohol-liposome interactions: a thermodynamic investigation. Prog. Anesth. Mech. vol. 6

80. C. Trandum, P. Westh, K. Jörgensen, and O. G. Mouritsen (1999) A calorimetric investigation of the interaction of short chain alcohols with unilamellar DMPC liposomes. J. Phys. Chem. B 103, pp. 4751-4756

81. P. Westh and C. Trandum (1999) Thermodynamics of alcohol-lipid bilayer interactions: application of a binding model. Biochim. Biophys. Acta 1421, pp. 261272

82. P. Westh, C. Trandum, and Y. Koga (2001) Binding of small alcohols to a lipid bilayer membrane: does the partitioning coefficient express the net affinity? Biophys. Chem. 89, pp. 53-63

83. T. Adalsteinsson and H. Yu (2000) Lipid lateral diffusion in multi-bilayers, and in monolayers at the air/water and heptane/water interfaces. Langmuir 16, pp. 9410-9413

84. L. Pauling (1960) The nature of the chemical bond. New York: Cornell University Press

85. O. G. Mouritsen and M. Blom (1984) Matress model of lipid-protein interactions in membranes. Biophys. J. 46, pp. 141-153

86. E. Sackmann (1984) Physical basis of trigger processes and membrane structure. In Biological Membranes vol. 5 (D. Chapman ed.), pp. 105-143, Academic Press, London

87. O. G. Mouritsen and M. M. Sperotto (1993) Thermodynamics of lipid-protein interactions in lipid membranes. In Thermodynamics of Membrane Receptors and 
Channels (M. Jackson, ed.), (Boca Raton, Florida), pp. 127-181, CRC Press, Inc.

88. F. Dumas, M. C. Lebrum, and J.-F. Tocanne (1999) Is the protein/lipid hydrophobic matching principle relevant to membrane organization and functions? FEBS Lett. 485, pp. 271-277

89. E. Strandberg, S. Özdirekcan, D. T. S. Rijkers, P. C. A. van der Wel, R. E. Koeppe II, R. M. J. Liskamp, and J. A. Killian (2004) Tilt angles of transmembrane model peptides in oriented and non-oriented lipid bilayers as determined by ${ }^{2} \mathrm{H}$ solid state NMR. Biophys. J. 86, pp. 3709-3721

90. R. B. M. Koehorst, R. B. Spruijt, F. J. Vergeldt, and M. A. Hemminga (2004) Lipid bilayer topology of the transmembrane $\alpha$-helix of M13 major coat protein and bilayer polarity profile by site-directed fluorescence spectroscopy. Biophys. J. 87, pp. $1445-1455$ 